ص ص[ [1 [1

معدلات هطول الأمطار وأثرها على إنتاجية محصول القمح دراسة

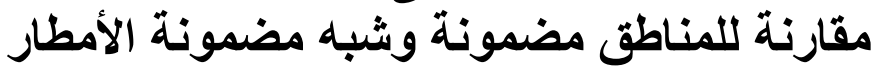
في محافظة نينوى
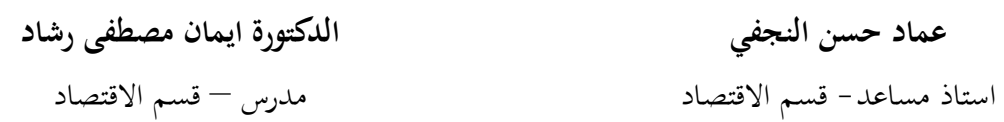

Ermostafa2000@yahoo.com

كلية الادارة والاقتصاد -جامعة الموصل

الكلمات المفتاحية: مناطق مضمونة الأمطار، معدلات هطول الأمطار، إنتاجية محصول القمح. 


\title{
Rainfall Levels and their Impact on Wheat Productivity: A Comparative Study between Rainfall Assured and Semi-Assured Regions in Nineveh
}

\author{
Emad H. AL-Najafee \\ Assistant Professor \\ Department of Economics \\ University of Mosul
}

\author{
Emaan M. Rashad (PhD) \\ Lecturer \\ Department of Eeconomics \\ University of Mosul \\ Ermostafa2000@yahoo.com
}

\begin{abstract}
The study aims at evaluating the impact of precipitation variance on the productivity of wheat in Nineveh under various rainfall levels during the wheat planting season 2008 2009. Large areas of land in this region depend on rain for their irrigation and thus affecting the income of a large number of farmers. The standard of living of those farmers approaches poverty during the dry seasons. This means that there is no strong correlation between the average rainfall and the production of wheat but between the distribution of rainfall patterns and the production a fact assured by the statistical distribution. The increase of rainfall in the lands which are classified as rainfall assured by $1 \%$ will lead to the increase in production by $29 \%$ while in the same level of increase the productivity will increase by $35 \%$ and $55 \%$ respectively under a statistical significant accepted level, while the variances in semi-assured land were not significant. Thus, the land use, for this purpose, must rely solely on rainfall, especially in the lands that are classified as rainfall assured, but complementary irrigation must also be used and in this case will increase the productivity per unit of land planted with wheat and may increase the well beings of these lands.
\end{abstract}

Key Words: Rainfall assured, Rainfall levels, Wheat Productivity.

المقدمة

يتميز النشاط الزراعي بحساسيته العالية للظروف الطبيعيـة المناخيـة وتقلباتهـا بدرجة إنة

كبيرة، إذ قد تؤثر رداءة الجو على بعض الأنشطة الاقتصادية الخدمبـة ولكن بشكل محدد

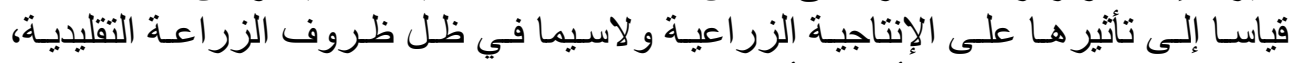

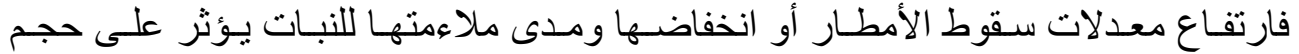

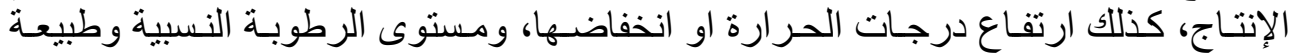

الرياح وشدتها، تؤثر جميعا على معدل إنتاجية المحصول، وقد شو هد في السنوات الثنلاث

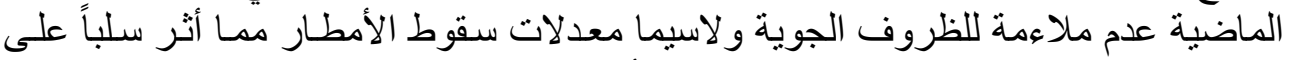

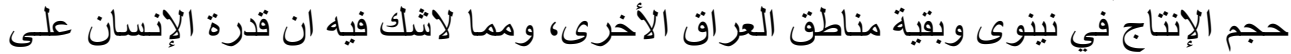

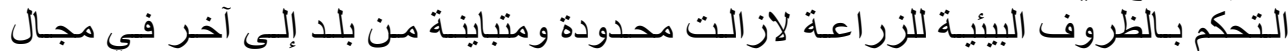

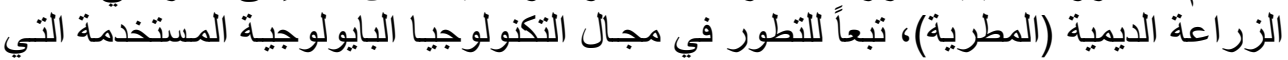
تتصف بخصوصبة المكان الذي تبتكر فيه، مايترتب على ذلك البطء الثديد لانتقال منثل هذه فئ

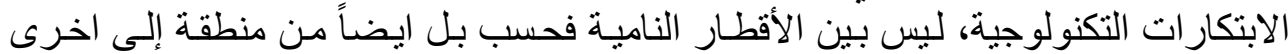

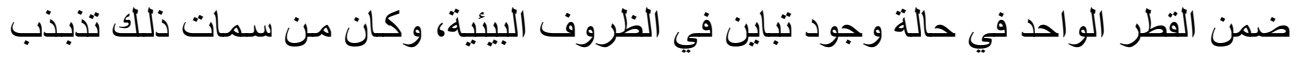
الإنتاج من محاصيل الحبوب الرئيسة (الحنطة و الثعير)، مايجعل مهمة تخطيط الإنتاج من 


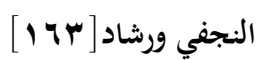

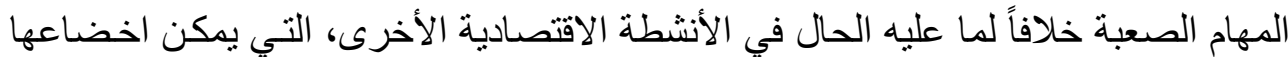

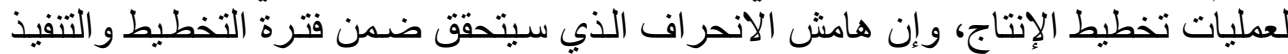

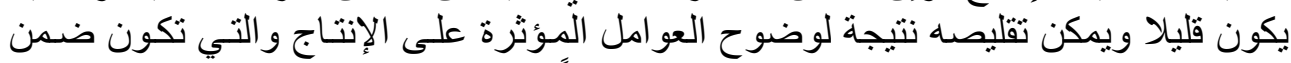

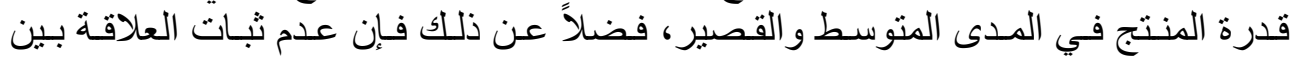

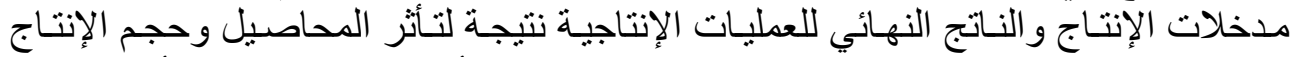

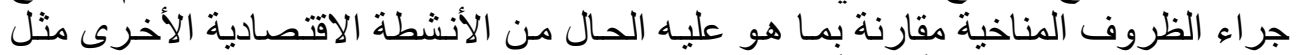

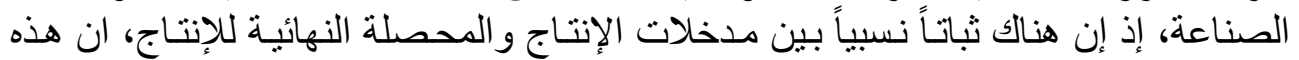

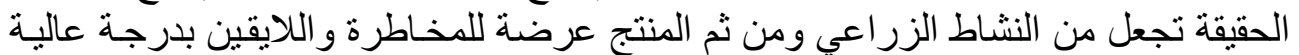

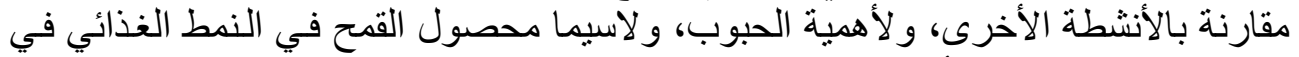

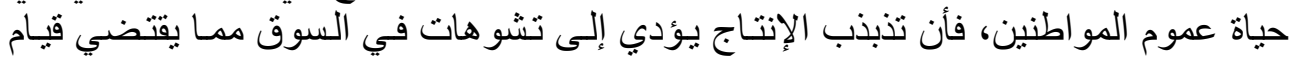

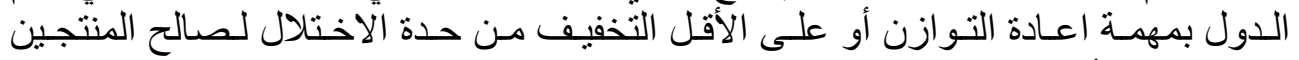

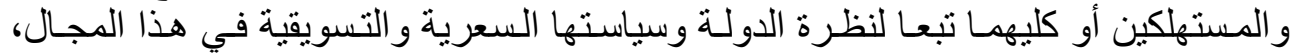
و تكاد تكون هذه المشكلة من بين المهام الأساسية التي توليها الدول بغض التهائا النظر عن فلسفتها

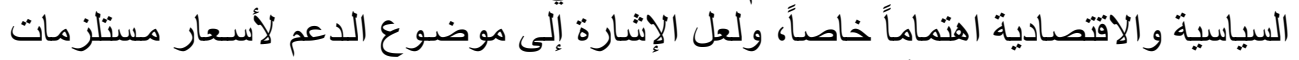

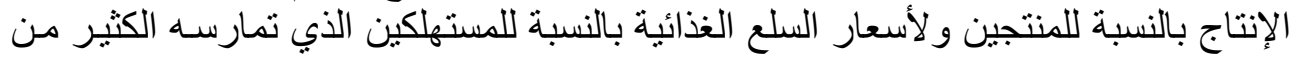
الدول يؤكد هذه الطروحات.

تأتي أهمية البحث بتز ايد الاهتمام في إنتاج و إنتاجية محاصيل الحبوب في العراق،

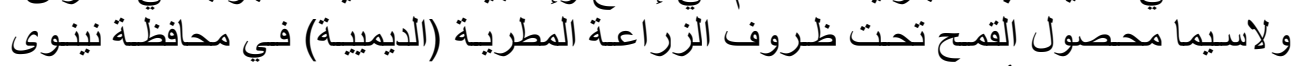

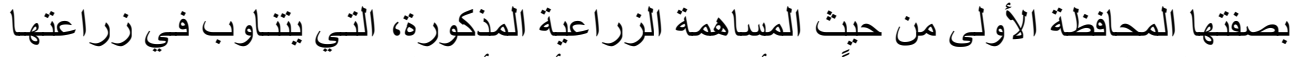

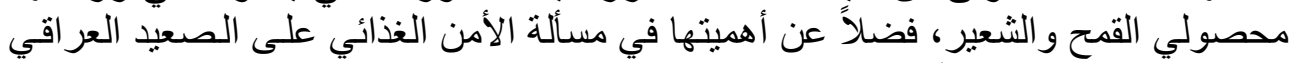

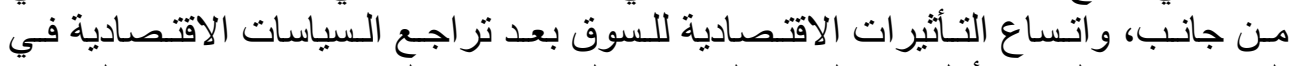

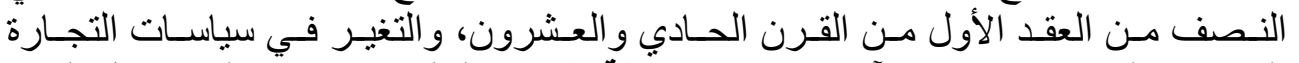

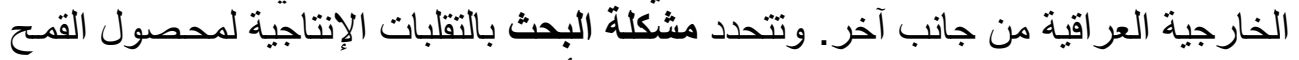

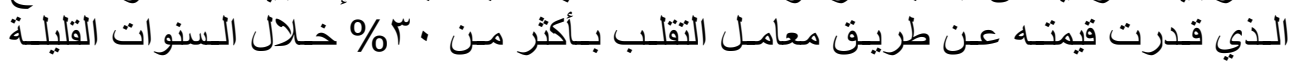

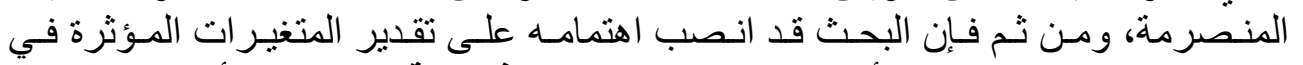

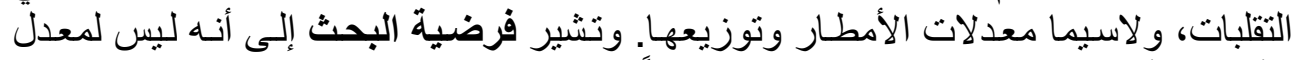

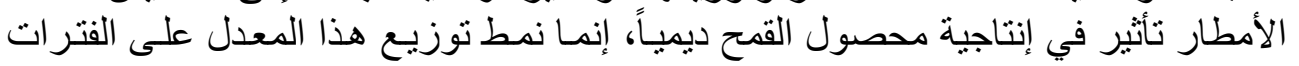

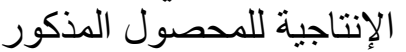

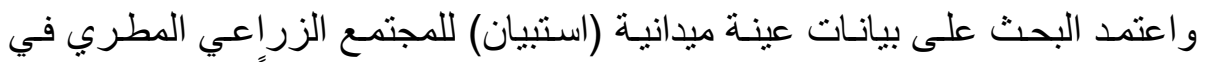

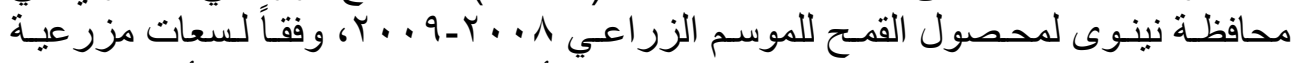

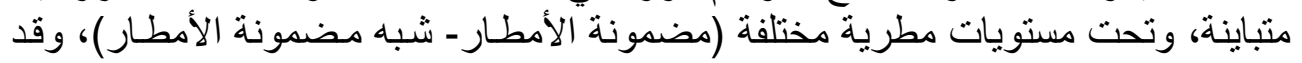

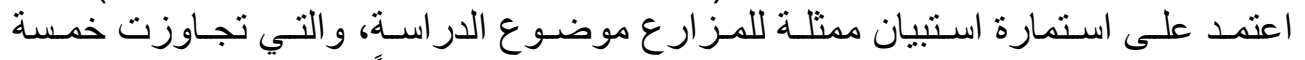

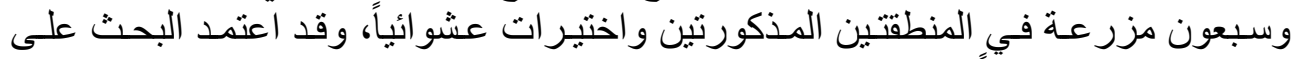
التحليل الوصفي فضلًً عن التحليل الكمي في اختبار الفرضية المذكورة.

التحليل والمناقشة

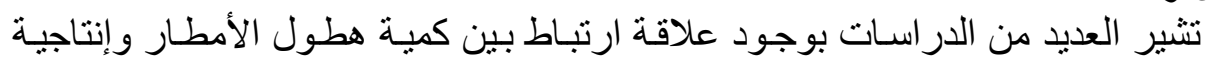

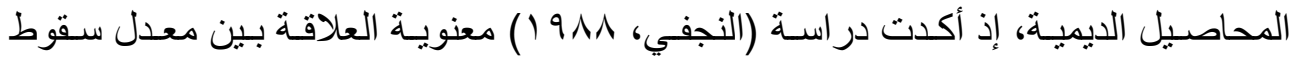

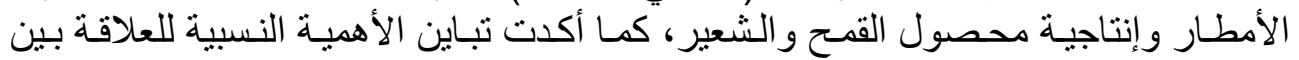




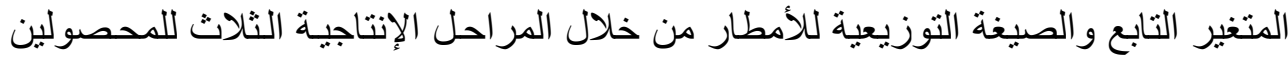

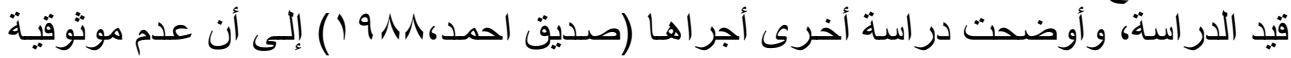

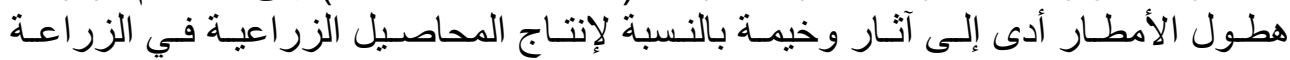

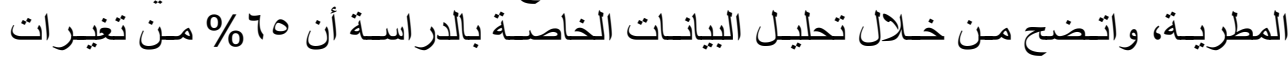

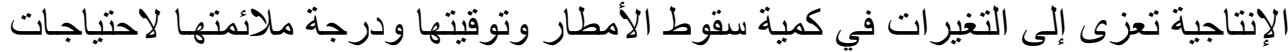

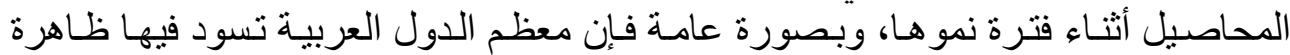

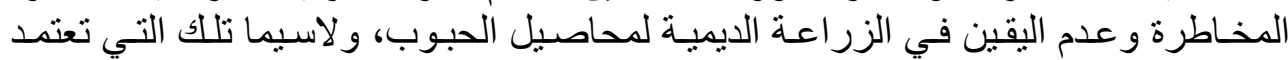

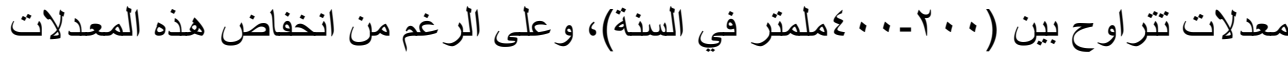

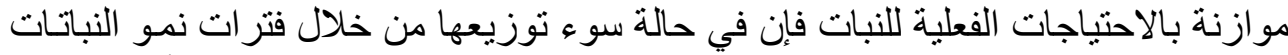

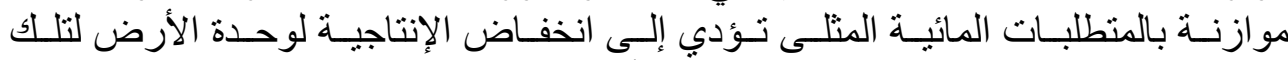

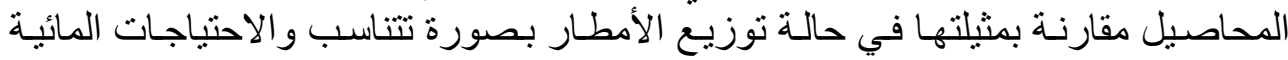

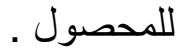

ومسن ثم فـإن قيـاس طبيعـة العلاقـة التوزيعيـة للأمطـار وتحديــ تأثير هـا في إنتاجيـة

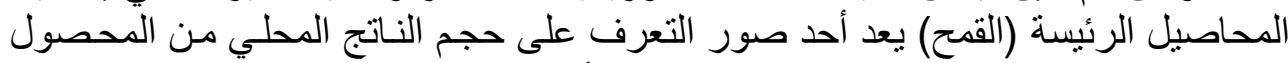

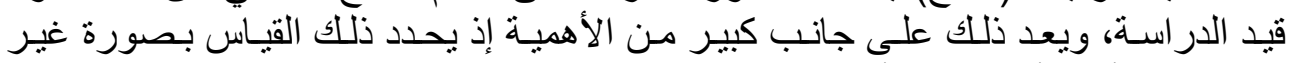

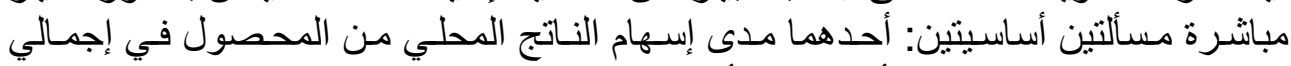

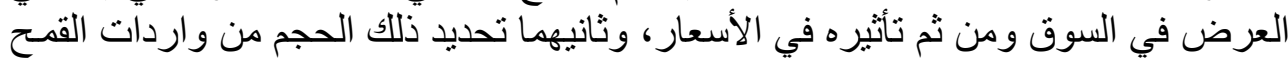

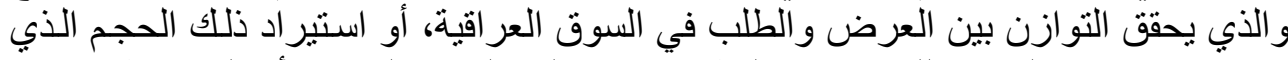

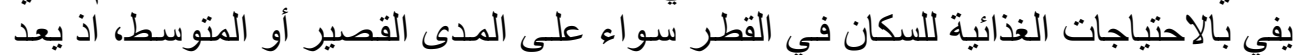

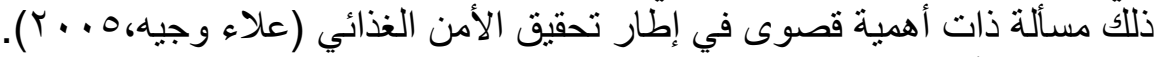

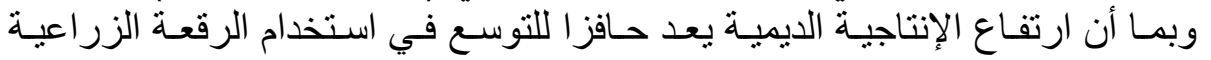

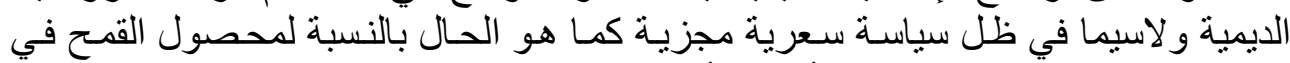

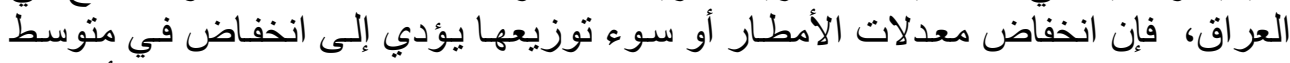

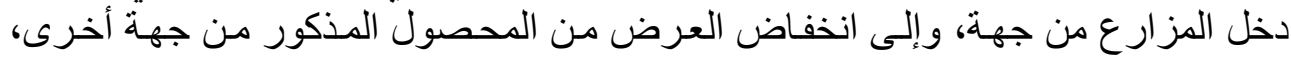

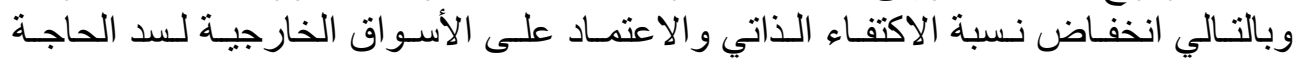
المحلية.

و ولى مستوى محافظة نينوى فإن البيئة الملائمة لزر اعة محصول القمح هي المناطق

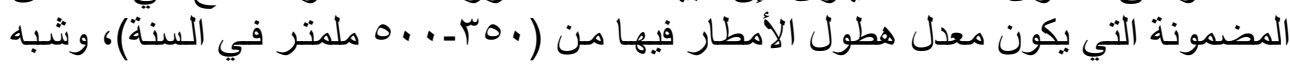

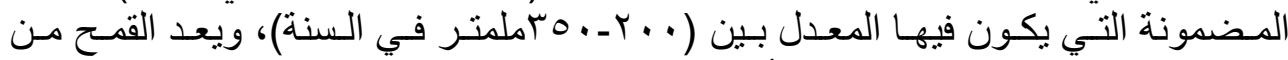

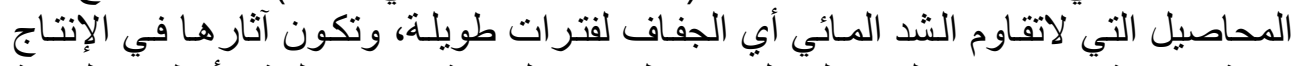

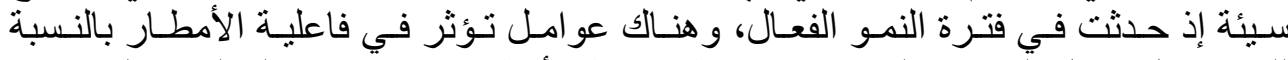

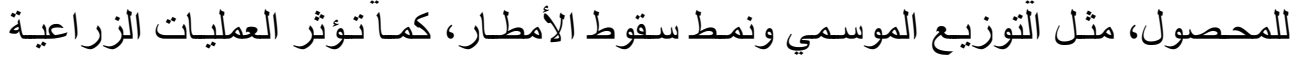

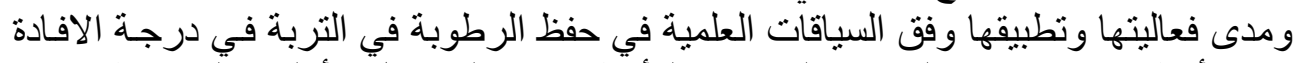

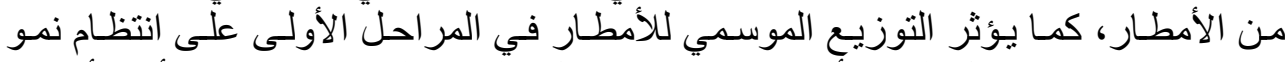

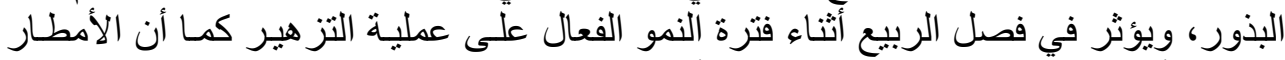

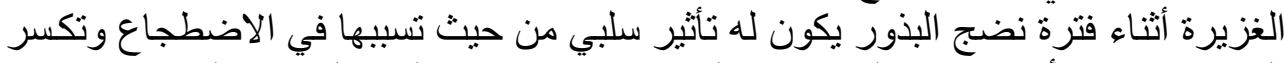

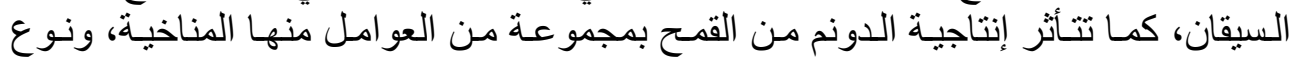

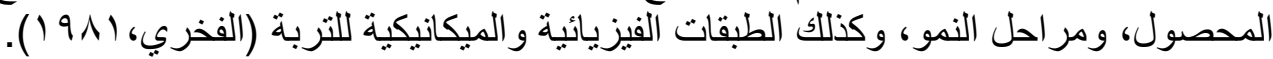




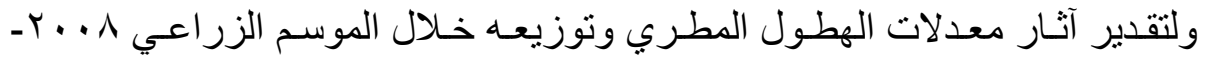

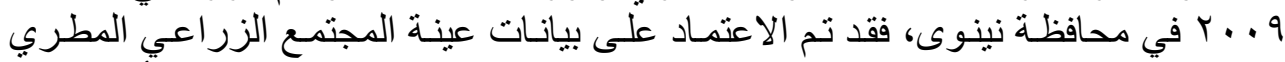

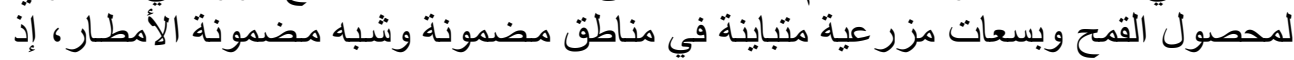

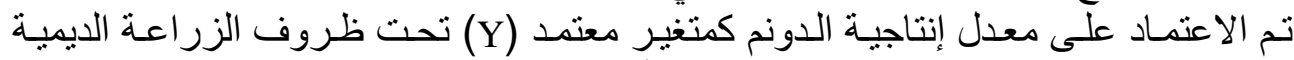

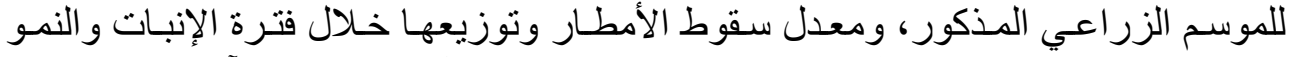
بوصفه مؤثر مفسر للمتغير المعتمد لنفس الموسم وتم التوصل إلى النتائج الآتية.

أولاً- المناطق المضمونة الأمطار

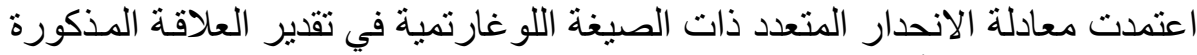

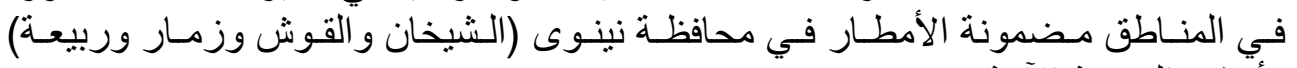

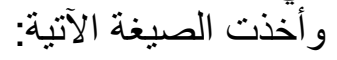

$$
\begin{aligned}
& \log \mathrm{Y}=0.237+0.294 \log \mathrm{X} 1+0.251 \log \mathrm{X} 2+0.554 \log \mathrm{X} 3 \\
& \mathrm{~T}=\quad \text { (6.64) } \\
& \mathrm{R}-\mathrm{Sq}=\mathbf{8 8 . 1 \%} \quad \mathrm{R}-\mathrm{Sq}(\operatorname{adj})=\mathbf{8 7 . 0 \%} \quad \mathrm{F}=\mathbf{7 9 . 2 3}
\end{aligned}
$$

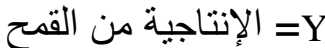

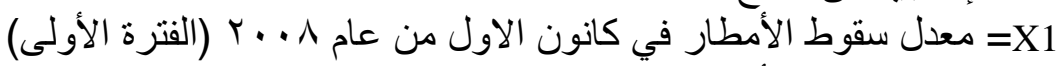

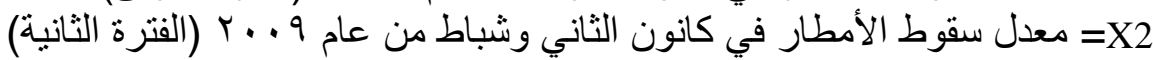

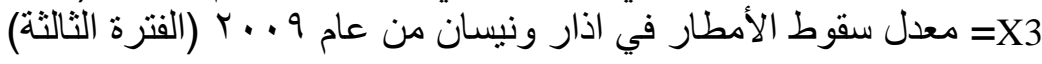

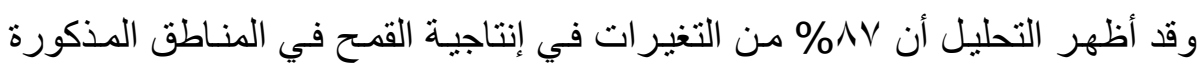

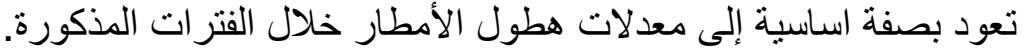

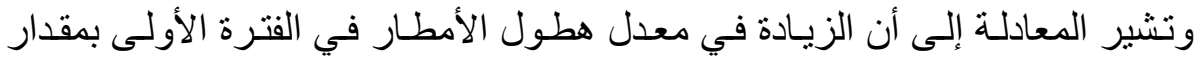

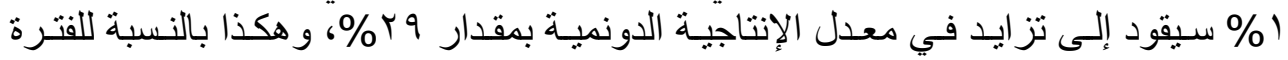

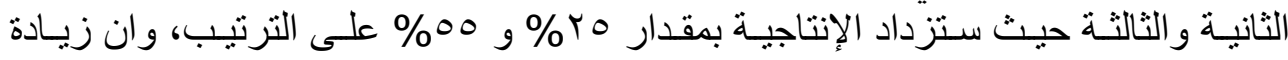

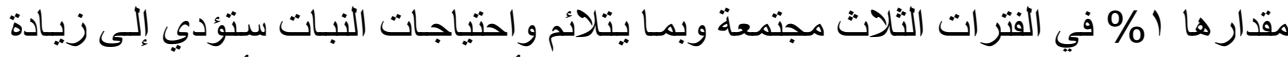

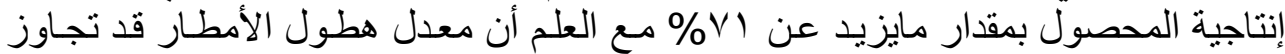

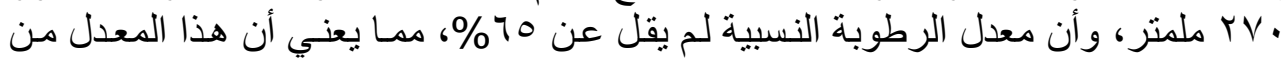

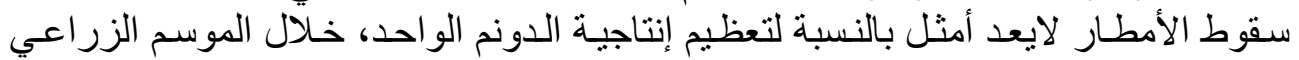

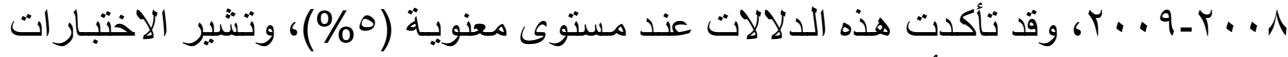
الإحصائية والقياسية إلى أن العلاقة الدالية ذات الثقات على عالية. أما على المستوى التجميعي للمناطق المذكورة آنفا فإن العلاقة الدالية بينهما أخذت التهات

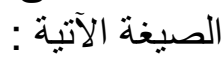

$$
\begin{aligned}
& \log \mathrm{Y}=-1.20+1.13 \log \mathrm{X} 1 \\
& T=\quad(12.34) \\
& \mathrm{R}-\mathrm{Sq}=\mathbf{8 1 . 7 \%} \quad \mathrm{R}-\mathrm{Sq}(\operatorname{adj})=\mathbf{8 1 . 2 \%} \quad \mathrm{F}=\mathbf{1 5 2}
\end{aligned}
$$


على الرغم من أن الاتجاه العام التجميعي لايعطي دلالة دقيقة عن سلوكية الإنتاجيـة المحصولية و لاسيما أن هذا الموسم قد اتصف بأنخفاض معدل هطول الأمطار على مستوى

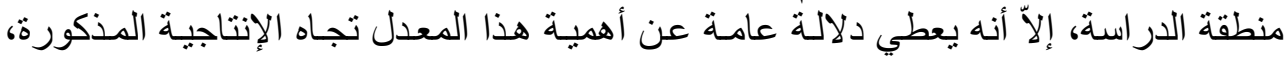

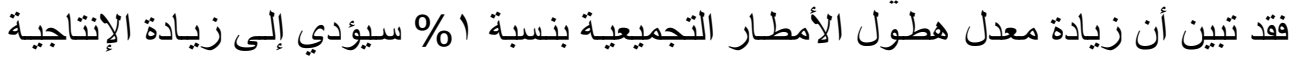

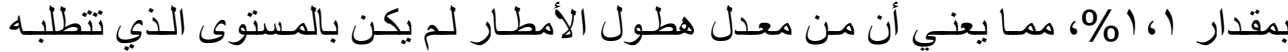

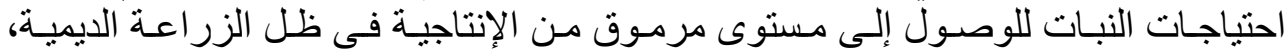

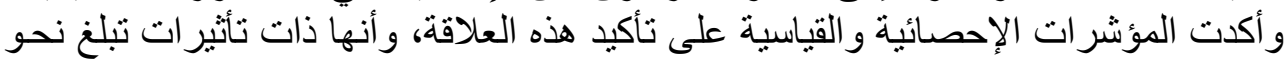

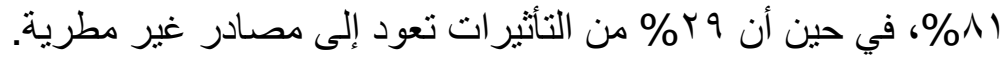

ثانياً. المناطق شبه مضمونة الأمطار

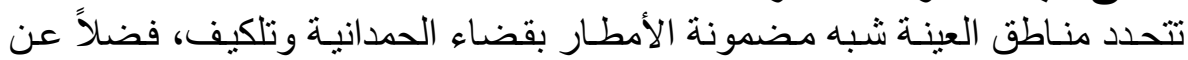

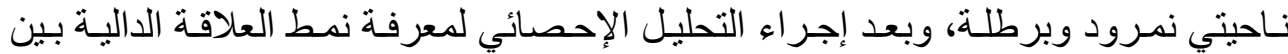

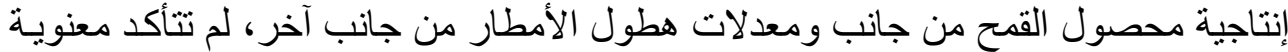

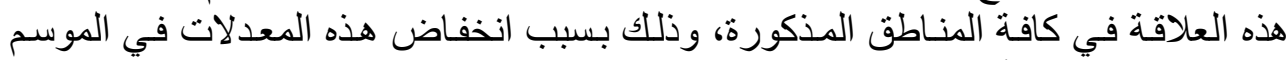

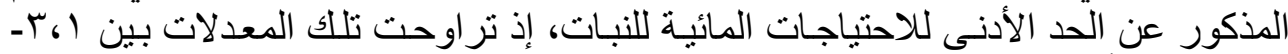

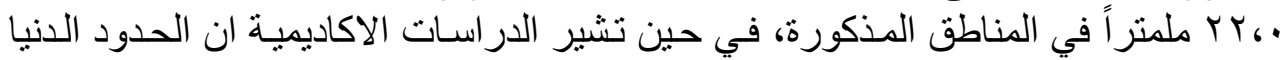

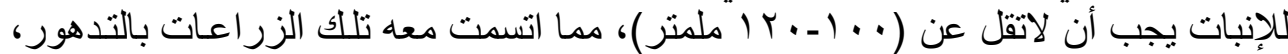

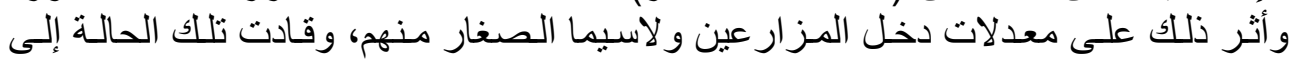

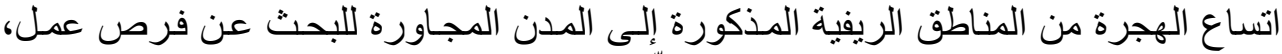

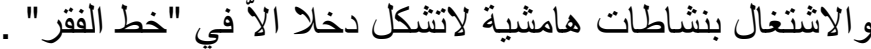

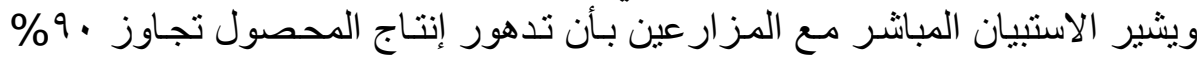

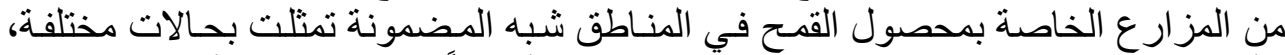

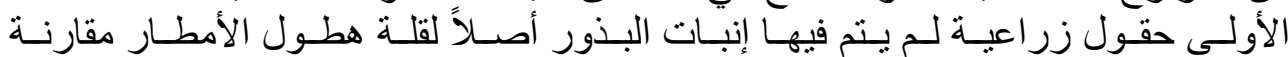

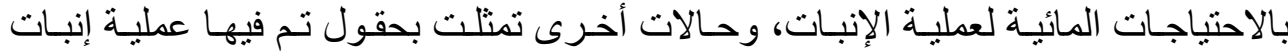

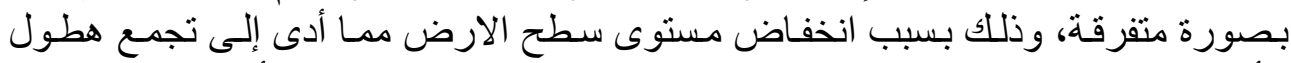

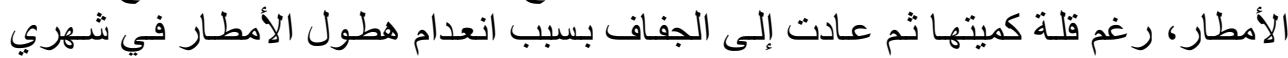

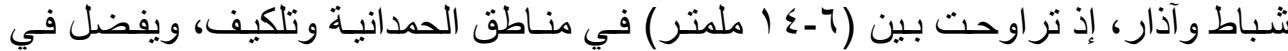

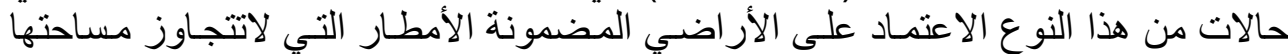

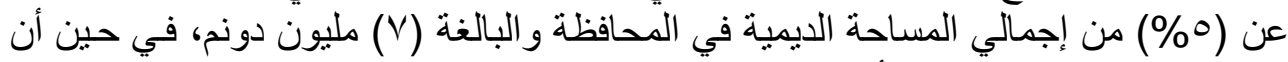

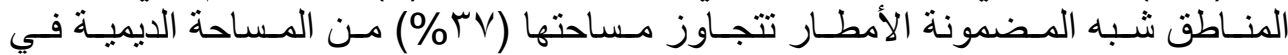

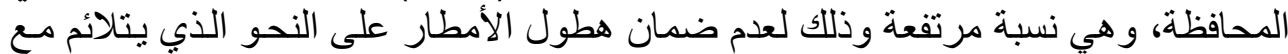

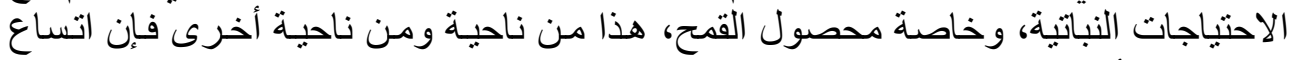

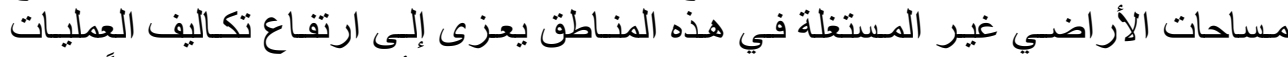

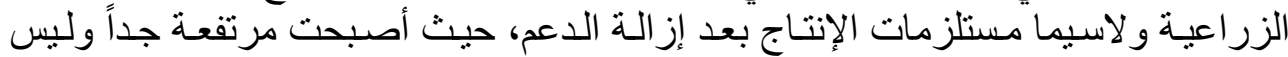

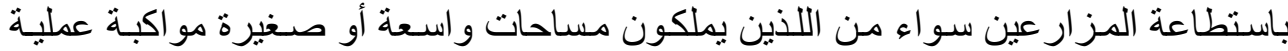

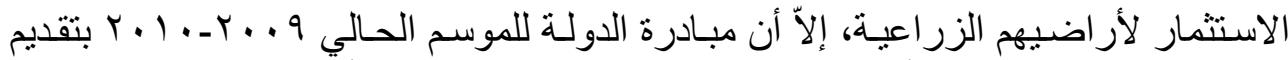

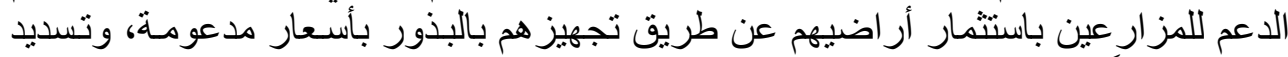

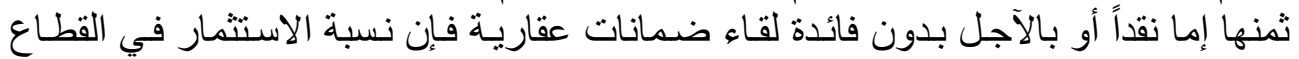




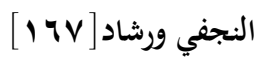

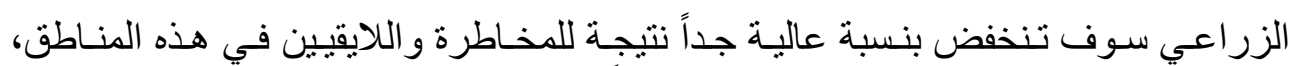
ويثير الدخطط 1 إلى نمط توزيع الأراضي وفقاً لأسلوب استخدامها في محافظة نينوى.
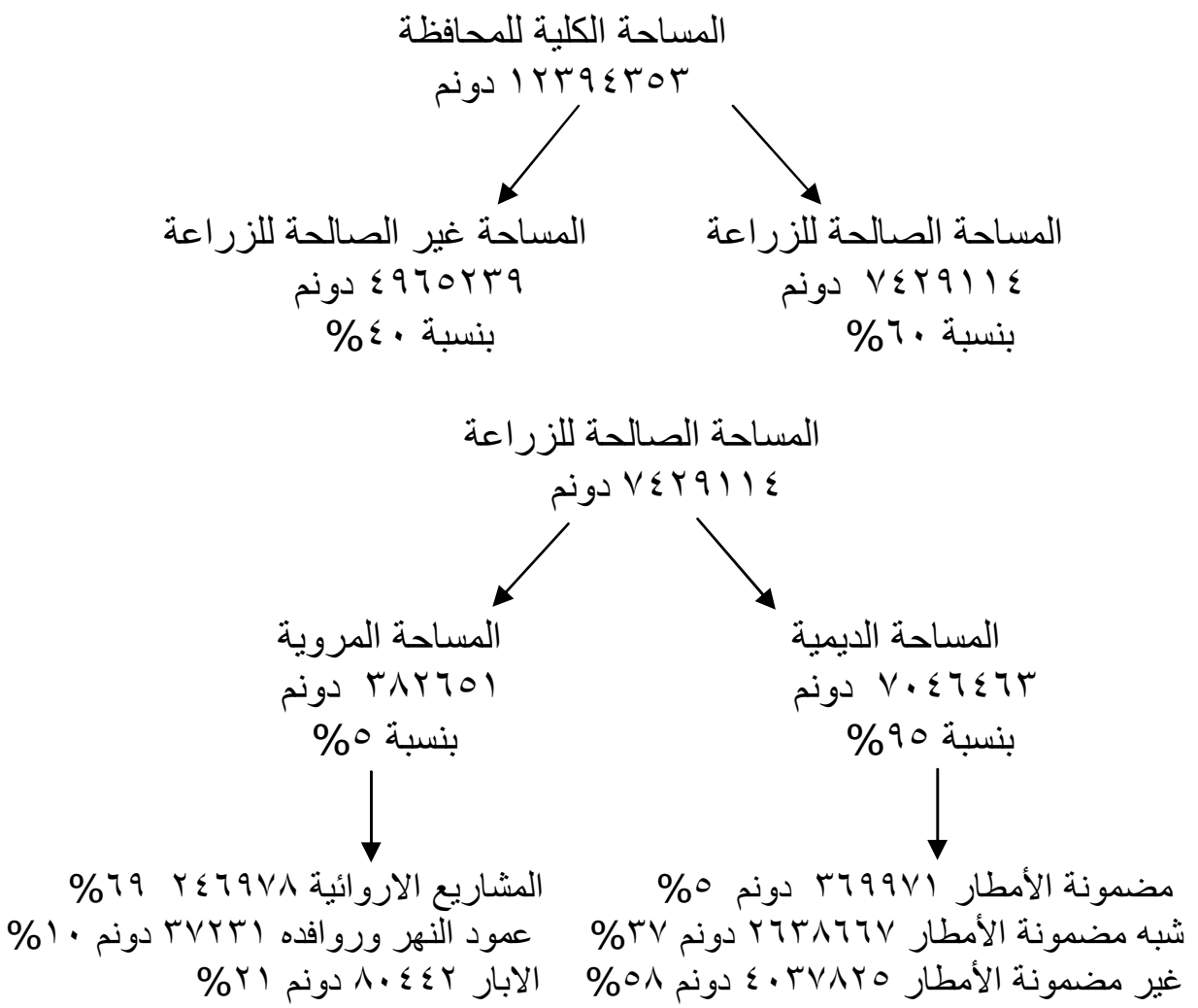

مخطط

توزيع استخدامات الأراضي الزراعية

المصدر: يوضح الثكل ا معدلات هطول الأمطار في مناطق العينـة للفترات الإنتاجية الثلاثتة في

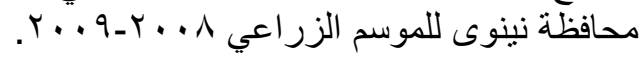




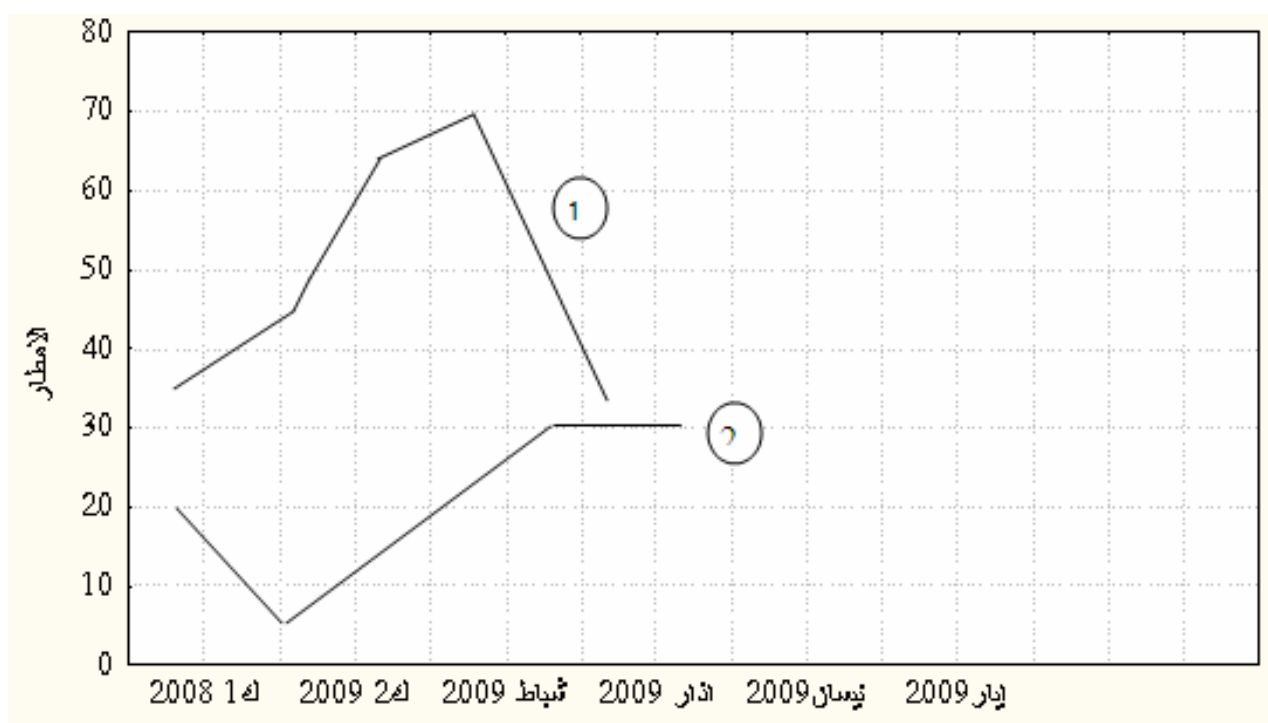

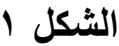

هطول الامطار بالملمتر للفترات الانتاجية الثلاثة

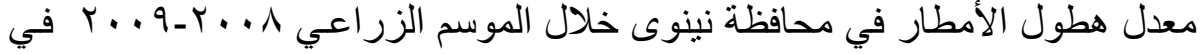

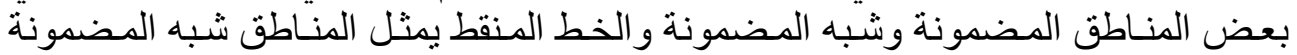
والخط بدون تنقيط يمثل المناطق المضمونة.

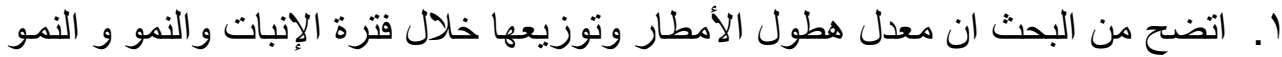

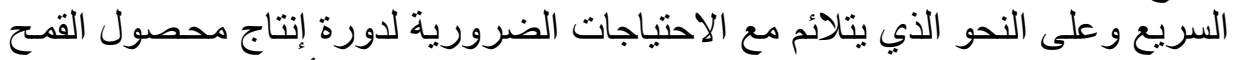

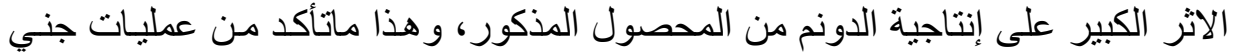

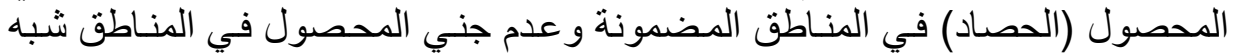

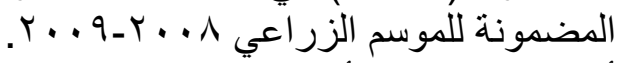

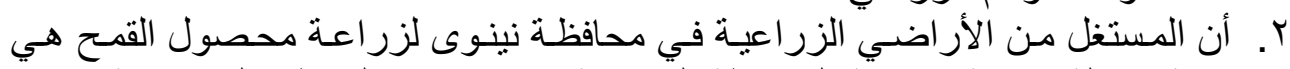

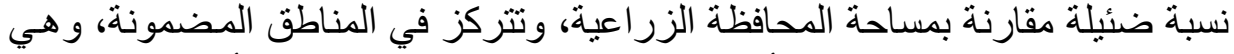

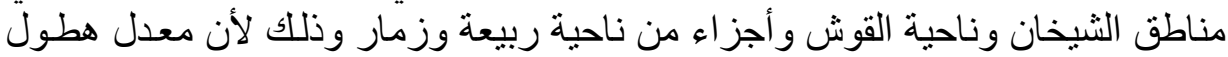

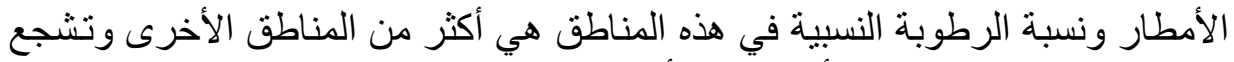

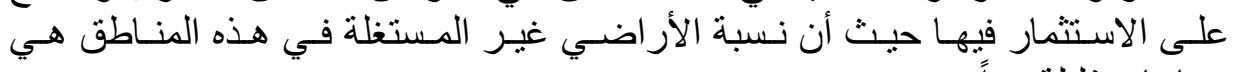

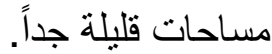

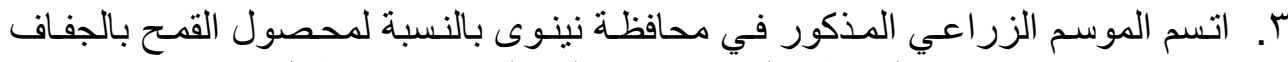

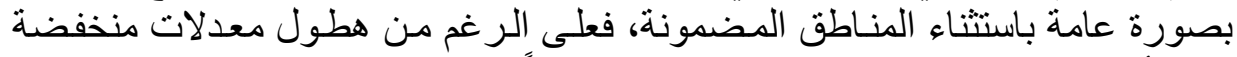

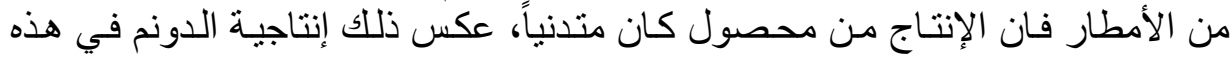

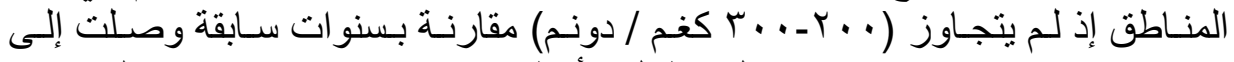

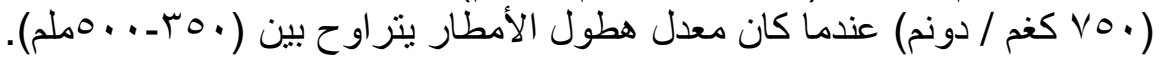




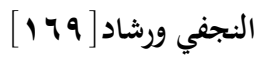

ع. لازال الطابع التقليدي هو السائد في تنفيذ العمليات الزراعيـة، ولاز الت أعداد كبيرة

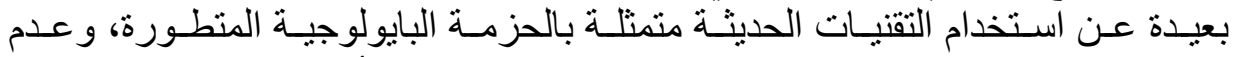

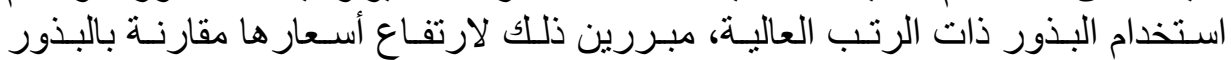

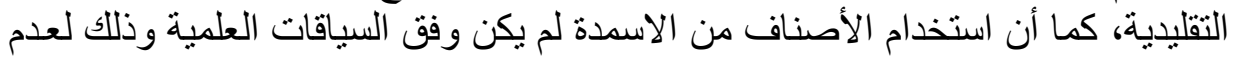

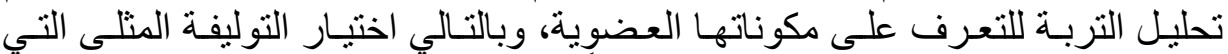

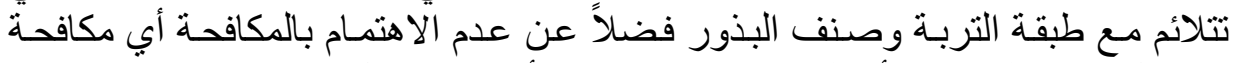

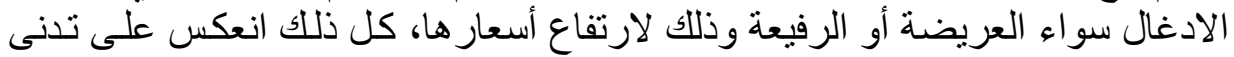

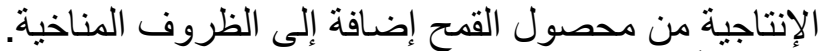

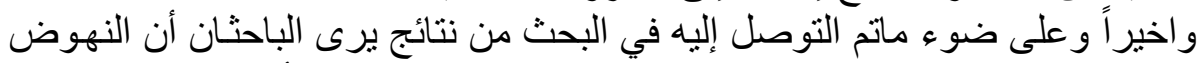

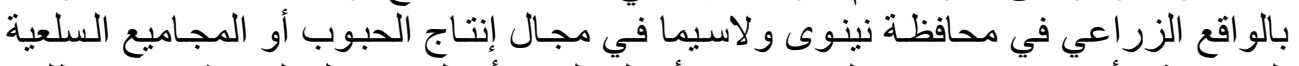

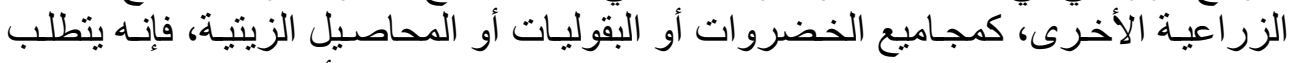

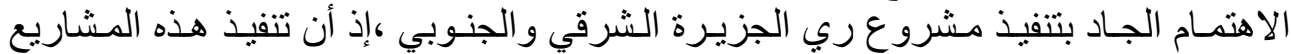

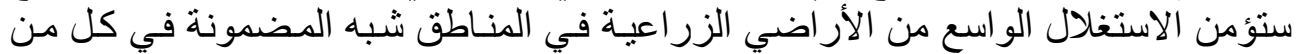

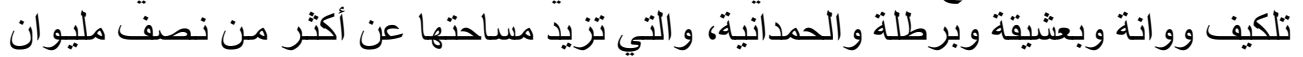

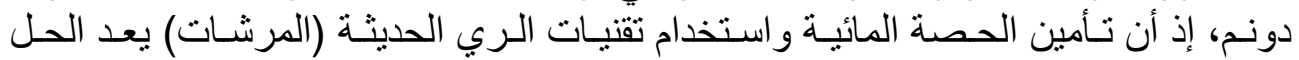

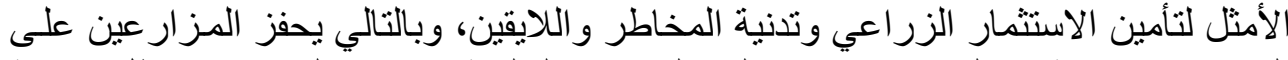

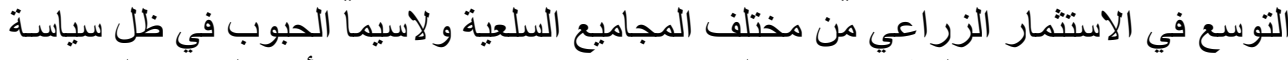

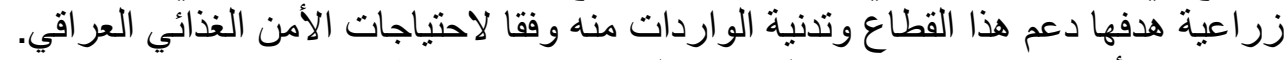

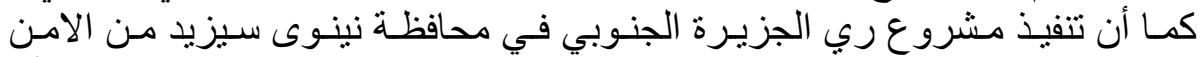

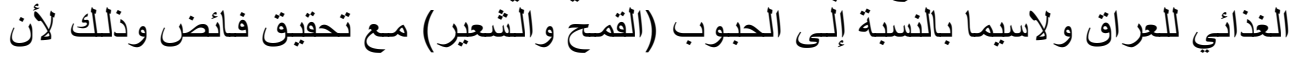

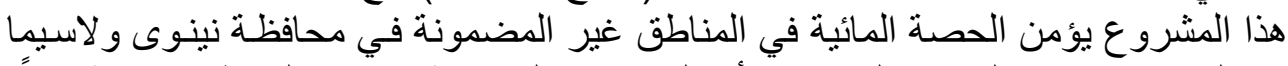

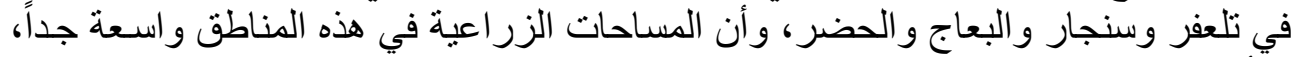

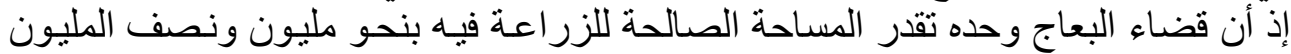
دونم، وكذلك الحال في بقية المناطق.

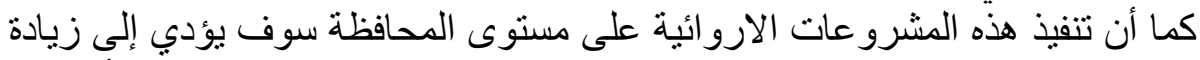

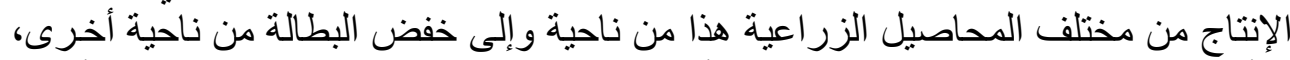

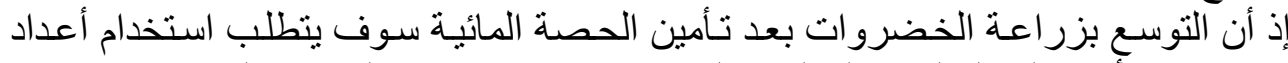

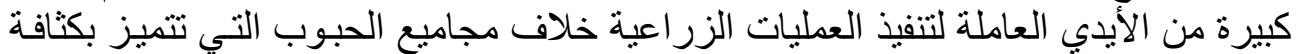
رأس المال في تنفيذ العمليات الزراعية.

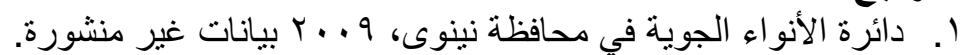

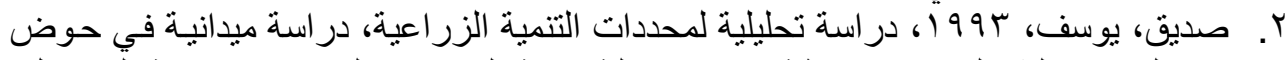

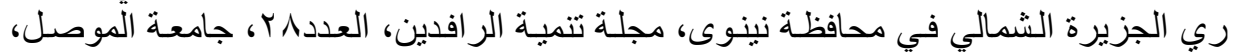

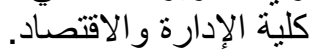

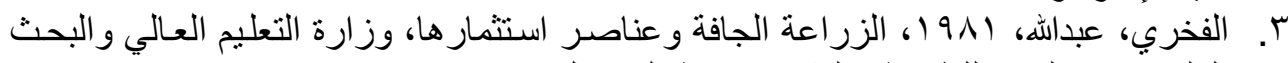

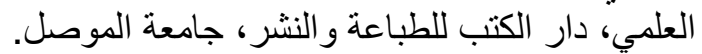

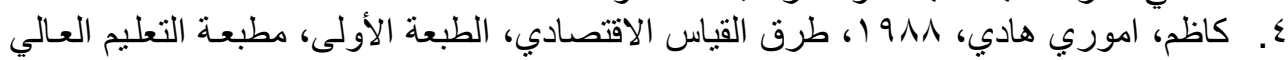

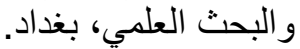

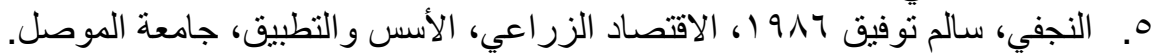




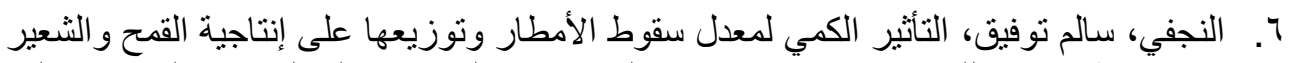

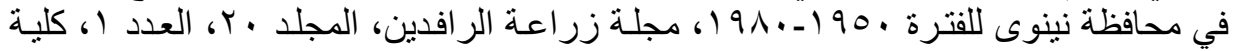

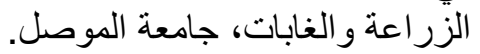

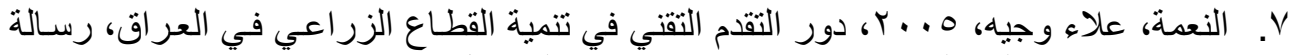

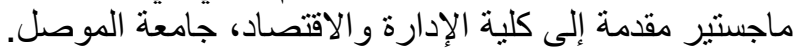

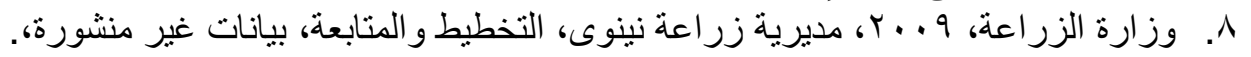

